\title{
UTILIZAÇÃO DE INFORMAÇÕES CONTÁBEIS E GERENCIAIS POR PARTE DOS EMPRESÁRIOS DE MICROS E PEQUENAS EMPRESAS
}

\author{
Cleonice Witt ${ }^{1}$ \\ Caroline Sulzbach Pletsch ${ }^{2}$ \\ Nelson Hein ${ }^{3}$ \\ Adriana Kroenke ${ }^{4}$
}

\begin{abstract}
RESUMO: O estudo tem como objetivo analisar as informações contábeis e gerenciais utilizadas pelos gestores de micros e pequenas empresas. Trata-se de um estudo descritivo, de levantamento e quantitativo. Compõe a amostra do estudo 73 micros e pequenas empresas sediadas no Vale do Itajaí/SC. Os dados foram coletados mediante aplicação de questionário com questões de múltipla escolha e escala Likert, sendo o tipo de amostragem por acessibilidade. Para análise de dados aplicou-se análise de correspondências múltiplas (ANACOR) utilizando o software LHStat 4.5. Os resultados indicam que o tempo de atuação da maioria das empresas é de 6 a 10 anos, atuam no comércio e possuem até 5 funcionários, sendo que os sistemas de controle de compras, pagamento de credores, contas a receber e gerenciamento do fluxo de caixa se destacaram como sendo os mais utilizados. Ainda, as empresas com menor tempo de mercado e com gestores que possuem educação continuada tendem a utilizar a contabilidade para fins gerenciais, enquanto que empresas com maior tempo de mercado e com pouca educação continuada, tendem a utilizar a contabilidade somente para fins fiscais. Esse estudo contribui para reforçar a importância da contabilidade gerencial para as microempresas/empresas de pequeno porte, pois dentre outras coisas, a sua utilização permite a elaboração de índices econômico-financeiros que auxiliam a tomada de decisão e por consequência propiciam a sobrevivência e crescimento da empresa.
\end{abstract}

Palavras-Chave: Informação. Contabilidade. Gestão. Micros e Pequenas Empresas.

\footnotetext{
${ }^{1}$ Doutoranda em Ciências Contábeis e Administração (FURB). Universidade do Contestado (UnC); Fundação Universidade Regional de Blumenau (FURB). Santa Catarina. Brasil. E-mail: cleonicewitt7@gmail.com

${ }^{2}$ Doutoranda em Ciências Contábeis e Administração (FURB). Universidade do Estado de Santa Catarina (UDESC); Fundação Universidade Regional de Blumenau (FURB). Santa Catarina. Brasil. E-mail: carol spletsch@yahoo.com.br

${ }^{3}$ Professor do Programa de Pós-graduação em administração e ciências contábeis da Fundação Universidade Regional de Blumenau (FURB). Santa Catarina. Brasil. E-mail: hein@furb.br

${ }^{4}$ Professora do Programa de Pós-graduação em administração e ciências contábeis da Fundação Universidade Regional de Blumenau (FURB). Santa Catarina. Brasil. E-mail: akroenke@furb.br
}

Ágora: R. Divulg. Cient., v. 23, n. 2, p. 73-94, jul./dez. 2018 (ISSNe 2237-9010) 


\title{
USE OF ACCOUNTING AND MANAGEMENT INFORMATION BY MICRO AND SMALL BUSINESS ENTREPRENEURS
}

\begin{abstract}
The purpose of this study is to analyze the accounting and management information used by managers of micro and small companies. This is a descriptive, survey and quantitative study. The study sample consisted of 73 micro and small companies based in the Itajaí Valley / SC. The data were collected through a questionnaire with questions of multiple choice and Likert scale, being the type of sampling by accessibility. For analysis of data, multiple correspondence analysis (ANACOR) was applied using the LHStat 4.5 software. The results indicate that the time of operation of most companies is from 6 to 10 years, they operate in the commerce and have up to 5 employees, being the systems of control of purchases, payment of creditors, accounts receivable and management of the cash flow were highlighted as being the most used. Also, companies with shorter time to market and managers with continuing education tend to use accounting for managerial purposes, while companies with longer market time and with little continuing education tend to use accounting for tax purposes only. This study contributes to reinforce the importance of managerial accounting for microenterprises / small businesses, since among other things, their use allows the elaboration of economic and financial indices that help decision making and, consequently, promote the survival and growth of company.
\end{abstract}

Keywords: Information. Accounting. Management. Micros and Small Business.

\section{INTRODUÇÃO}

Todo empreendimento independentemente da origem do capital, do porte e da sua natureza jurídica necessita de pessoas que executem as atividades com o intuito de atingir o fim a que se propõe. Na condução do negócio essas pessoas necessitam de informações de diversas naturezas: econômica, social, ambiental, empresarial, tributária, legal entre outras, o que gera a necessidade de construir um arcabouço de informações capaz de subsidiar a tomada de decisões. Na construção dessa estrutura informacional se destaca a contabilidade, pois o seu objetivo é fornecer informações aos seus usuários propiciando uma base segura para a tomada de decisões (BEATTY; LIAO, 2014).

Nesse sentido a informação contábil fornece conhecimento para o gestor se preparar para ações e decisões futuras, tornando visíveis atividades que não são visíveis nas suas rotinas diárias e fornecendo uma perspectiva quantitativa sobre o seu trabalho (HALL, 2010). Esse papel da contabilidade é desempenhado através do 
provimento de dados sob a forma de relatórios e análises (SPRINKLE, 2003), proporcionando uma avaliação global das tendências e resultados de todas as perturbações e ações que ocorreram (VAN DER VEEKEN; WOUTERS, 2002).

Se, por um lado, a informação contábil se apresenta como uma importante base para a tomada de decisões, por outro lado se observa que muitos usuários não conhecem os termos técnicos empregados na contabilidade para evidenciação dos eventos (MOREIRA, 2013). Isso ocorre principalmente nas pequenas e médias empresas, pois normalmente elas não dispõem de pessoal capacitado para interpretar essas informações. Essa falta de habilidade dos gestores das micros e pequenas empresas em entender e interpretar a produção contabilística foi uma dificuldade apontada em alguns estudos (SHELDON, 1994; ALBUQUERQUE, 2004; CANECA, 2009; BERNARDES, 2011; SANTOS, 2016).

No entanto a ausência de utilização dessas informações pode ser em decorrência da falta de dados úteis para a tomada de decisões, ou seja, os contadores dessas empresas muitas vezes se limitam a prestar serviços de natureza fiscal ou ainda os gestores desconhecem os serviços adicionais que a contabilidade pode oferecer. Isso ocorre devido à influência fiscal nas informações prestadas pela contabilidade, ficando relegado ao segundo plano as informações que são base para a gestão do negócio (STROEHER, 2005).

Diante do exposto a questão que norteia o estudo é: Quais são as informações contábeis e gerenciais utilizadas pelos gestores de micros e pequenas empresas? Nesta perspectiva tem-se como objetivo analisar as informações contábeis e gerenciais utilizadas pelos gestores de micros e pequenas empresas. Desta forma o estudo se justifica pela quantidade de micros e pequenas empresas no Brasil e pela contribuição econômica que esse segmento traz. Para os profissionais contábeis é uma oportunidade para discutr a comunicação entre o contador e o gestor das micros e pequenas empresas. No campo acadêmico a importância do assunto é reconhecida pelos inúmeros trabalhos que estudaram as micros e pequenas empresas, dentre eles os estudos de Moreira et al. (2013) que constatou que a contabilidade é vista como mera executora das informações fiscais e trabalhistas e os gestores não percebem a importância da informação contábil; Lucena, Vasconcelos e Marcelino (2015) identificaram que as micros e pequenas empresas tendem a realizar controles de estoques, de contas a receber, a pagar e de vendas; Kos et al. (2014) constataram 
que os gestores das micros e pequenas empresas recebem parte das informações contábeis, não as compreendem, porém as usam como subsídio em seu processo decisório, dentro do limite de sua compreensão; Santos et al. (2014) concluíram que dentre os instrumentos da contabilidade gerencial oferecidos pelo contador predominam as demonstrações contábeis e o planejamento tributário. Ainda como antecedente de pesquisa o SEBRAE em 2007 apontou que 40\% dos microempresários/pequenos empresários vem buscando auxílio dos contadores para o gerenciamento de sua empresa e por consequência os contadores devem prover esses empreendedores de informações capazes de suprir as suas necessidades de gerenciamento. Assim sendo, este estudo visa contribuir com a comunidade contábil provendo informações no que tange às necessidades dos gestores em relação às informações contábeis e assim possibilitar à esses profissionais adequarem a forma de apresentação dessas informações às reais necessidades dos usuários.

\section{REFERENCIAL TEÓRICO}

\subsection{INFORMAÇÃO CONTÁBIL E GERENCIAL}

A estrutura contábil de uma empresa permite o fornecimento de diversas informações como: previsão de vendas, necessidades de caixa, controles de despesas financeiras, controle dos prazos de vendas e compras (LUCENA, 2004); controle financeiro, de clientes e fornecedores, gestão do capital de giro, controle de estoque, gestão fiscal (UENO; CASA NOVA, 2006); controle de despesas, análise da margem de lucro, controle do imobilizado (OLEIRO; DAMEDA; VICTOR, 2007); controle da folha de pagamento, de tributos e contribuições, do contas a pagar, cálculo do lucro, depreciação e saldo de caixa (CANECA, 2008).

Denota-se assim que a contabilidade abarca informações de diversos setores da empresa, sendo que ela só agregará valor para a tomada de decisões se for capaz de proteger os ativos e o patrimônio da empresa, medir o desempenho das atividades executadas e elaborar informações confiáveis para os usuários (CAMELO; CAMELO; PAULINO, 2006). Da mesma maneira a informação gerencial se apresenta como fundamental para fornecer base aos gestores no momento da tomada de decisões, assim a informação gerencial tem como principal escopo os gestores dentro da 
organização, estando voltada exclusivamente para a administração da empresa para fornecer conhecimento que se encaixe de maneira efetiva no modelo decisório do administrador (FERNANDES; KLANN; FIGUEREDO, 2011).

\subsection{MICROS E PEQUENAS EMPRESAS}

Micros e pequenas empresas cada vez mais representam um papel importante na economia de muitos países, por esse motivo os governos focam o desenvolvimento desse segmento para promover o crescimento econômico (FATOKI, 2012). A contribuição dessas empresas é maior nas economias em desenvolvimento (AHMAD; MOHAMED, 2015), sendo consideradas parte integrante do desenvolvimento econômico do país (ZABRI, 2012). Devido à essa importância vários estudos tem como objeto as micros e pequenas empresas, no âmbito internacional (MUNGARAY; RAMÍREZ, 2007; TUNG; AYCAN, 2008; PETRUS, 2009; ABOR; QUARTEY, 2010; NAQVI, 2011; HOTHO, 2011; MANO et al., 2012; COWLING;LIU; LEDGER, 2012; GUPTA; SEETHARAMAN; RAJ, 2013; TUNDUI; TUNDUI, 2013; DAI; IVANOV; COLE, 2017; SHIBIA; BARAKO, 2017; SAES; HOURNEAUX JUNIOR, 2018; ALMEIDA, 2018) e nacional (HALL et al., 2012; BARBOZA; FONSECA; RAMALHEIRO, 2017; COSTA, 2018; PEREIRA, 2018; VASCONCELOS; OLIVEIRA, 2018; BARBOSA; FERREIRA, 2018; OTTONICAR; BASSETTO NASCIMENTO, 2018; PASCUCI; ALVES, 2018; FIEK; LOOSE, 2018).

No Brasil a micro e a pequena empresa são classificadas por dois critérios, o SEBRAE utiliza o critério de número de empregados e o Banco Nacional do Desenvolvimento (BNDES) e a legislação fiscal adotam a receita bruta como parâmetro. Há que se destacar ainda que além dos critérios acima, as agências de crédito e de financiamento estabelecem seus próprios critérios segundo o montante e as finalidades dos auxílios financeiros, visto que se preocupam com as garantias reais que as microempresas/empresas de pequeno porte podem oferecer, por isso se baseiam mais na situação patrimonial do que nas potencialidades da empresa refletidas por indicadores operacionais (LEONE et al., 1991).

Segundo Ângulo et al. (2010) essas empresas possuem limitada capacidade de geração de riqueza, embora evidências empíricas indicam que esse segmento não irá desaparecer, pois está se destacando cada vez mais no cenário empresarial 
Utilização de informações contábeis e gerenciais por parte dos empresários de micros e pequenas empresas

devido a sua contribuição ao emprego, sua flexibilidade e capacidade de inovação. Desta forma se destaca que as micros e pequenas empresas possuem características positivas, porém relutam em profissionalizar a administração, o que pode acarretar em seu fechamento prematuro (SEMLER, 1988).

\section{PROCEDIMENTOS METODOLÓGICOS}

A pesquisa classifica-se como descritiva, de levantamento e quantitativa. A população do estudo são as Micros e Pequenas Empresas do Vale do Itajaí/SC. Responderam ao questionário os proprietários das empresas e funcionários que participam da gestão, totalizando 73 questionários respondidos, os quais referem-se a amostra do estudo. Foi considerada a classificação fiscal para micros e pequenas empresas, ou seja, com base na receita bruta. No Quadro 2 apresenta-se o constructo do estudo.

Quadro 1 - Constructo do estudo

\begin{tabular}{|c|c|c|}
\hline Categoria & $x^{2}+x^{2}$ & Autores \\
\hline $\begin{array}{l}\text { Caracterização } \\
\text { da empresa e } \\
\text { dos } \\
\text { respondentes }\end{array}$ & $\begin{array}{l}\text { Tempo que a empresa existe no mercado } \\
\text { Setor da empresa } \\
\text { Quantidade de funcionários } \\
\text { Faixa Etária } \\
\text { Escolaridade }\end{array}$ & - \\
\hline $\begin{array}{l}\text { Informações } \\
\text { Contábeis e } \\
\text { Gerenciais }\end{array}$ & $\begin{array}{l}\text { Realização de cursos de gestão por ano } \\
\text { Relatórios gerenciais recebidos da } \\
\text { Contabilidade } \\
\text { Utiliza a contabilidade de apoio a gestão } \\
\text { Área em que a informação contábil é mais útil } \\
\text { Recursos utilizados para tomada de decisão }\end{array}$ & Moreira et al. (2013) \\
\hline $\begin{array}{l}\text { Informações } \\
\text { de } \\
\text { gerenciamento } \\
\text { e sistemas de } \\
\text { controle }\end{array}$ & $\begin{array}{l}\text { Objetivos estratégicos } \\
\text { Gerenciamento de fluxo de caixa } \\
\text { Previsões de fluxo de caixa } \\
\text { Controles de compras } \\
\text { Controles de pagamento de credores } \\
\text { Controle de estoque } \\
\text { Controle de devedores } \\
\text { Gestão de financiamento } \\
\text { Margem e gerenciamento de custos } \\
\text { Folhas de tempo de trabalho e custos de } \\
\text { trabalho } \\
\text { Preços e mark-ups } \\
\text { Controle de margem bruta } \\
\text { Análise de equilíbrio } \\
\text { Sensibilidade de custo-volume-lucro } \\
\text { Orçamento básico } \\
\text { Contas de lucros e perdas } \\
\text { Balanço patrimonial } \\
\text { Demonstração do Resultado do Exercício }\end{array}$ & Perren e Grant (2000) \\
\hline
\end{tabular}

Ágora: R. Divulg. Cient., v. 23, n. 2, p. 73-94, jul./dez. 2018 (ISSNe 2237-9010) 


\begin{tabular}{|l|l|l|}
\hline & Monitoramento da satisfação do cliente & \\
\hline $\begin{array}{l}\text { Competências } \\
\text { Gerenciais }\end{array}$ & Competência Sociais & Bündchen, Rossetto e Silva \\
& Competências Técnico Profissionais & (2008), \\
\hline
\end{tabular}

Fonte: Dados da pesquisa.

Foi realizado pré-teste do questionário com cinco proprietários de empresa, para verificar a clareza e entendimento das questões, sendo que esse procedimento culminou com a alteração da redação de duas questões do questionário. O questionário estruturado com questões de múltipla escolha e em escala Likert foi aplicado por meio do link do Google formulário e também entregue impresso aos possíveis respondentes. O link foi enviado para os escritórios de contabilidade para envio aos seus clientes e o questionário impresso foi entregue diretamente nas empresas, ficando caracterizada a amostragem por acessibilidade.

Após finalizada a coleta dos dados, as respostas foram transportadas para a planilha do Excel. Utilizou-se da estatística descritiva e análise de correspondências múltiplas (ANACOR) para a análise dos dados. A Anacor foi utilizada para verificar as características dos respondentes e das empresas que usam e não usam as informações contábeis e gerenciais para a tomada de decisão.

A técnica ANACOR tem como foco a verificação da associação entre variáveis que são categóricas e que geram tabelas de contingência (MINGOTI, 2005). Quando o número de variáveis e categorias não é grande, usa-se o teste Qui-quadrado para avaliar se a informação contida nas linhas das tabelas é independente ou não das informações contidas nas colunas. Quando o conjunto de dados é maior, como é o caso deste trabalho, o uso da técnica Qui-quadrado fica limitada, pois existe a necessidade de relacionar um grande número de variáveis e categorias. Nestes casos, uma opção é a análise de correspondências (ANACOR) (GAMBIRAGE, et al., 2017).

Fávero et al. (2009 p. 271) contribui afirmando que:

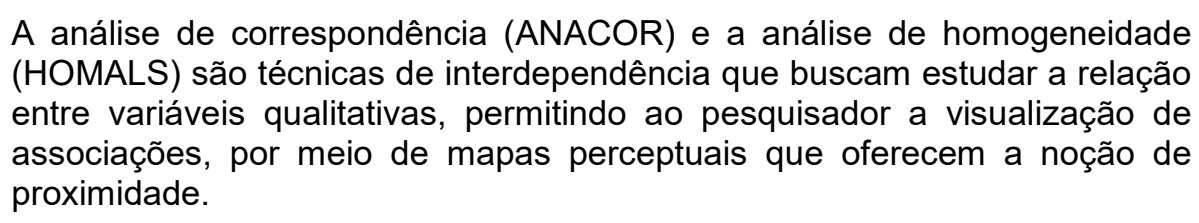

Mais adiante agrega "a ANACOR é uma técnica que exibe as associações entre um conjunto de variáveis categóricas não métricas em um mapa perceptual, Ágora: R. Divulg. Cient., v. 23, n. 2, p. 73-94, jul./dez. 2018 (ISSNe 2237-9010) 
permitindo desta maneira, um exame visual de qualquer padrão ou estrutura de dados" (FÁVERO et al., 2009, p. 272).

Matematicamente, parte-se de uma tabela de contingência conforme Tabela 1:

Tabela 1 - Tabela de Contingência

\begin{tabular}{|c|c|c|c|c|c|c|c|}
\hline & \multicolumn{7}{|c|}{ Variável Y } \\
\hline \multirow{6}{*}{ Variável X } & & 1 & 2 & 3 & $\ldots$ & $q$ & Total \\
\hline & 1 & $\mathrm{n}_{11}$ & $\mathrm{n}_{12}$ & $\mathrm{n}_{13}$ & $\ldots$ & $\mathrm{n}_{1 \mathrm{q}}$ & $\mathrm{n}_{1}$ \\
\hline & 2 & $\mathrm{n}_{21}$ & $\mathrm{n}_{22}$ & $\mathrm{n}_{23}$ & $\ldots$ & $\mathrm{n}_{2 \mathrm{q}}$ & $\mathrm{n}_{2}$. \\
\hline & 3 & $\mathrm{n}_{31}$ & $\mathrm{n}_{32}$ & $\mathrm{n}_{33}$ & $\ldots$ & $n_{3 q}$ & $\mathrm{n}_{3}$. \\
\hline & $\ldots$ & $\ldots$ & $\ldots$ & $\ldots$ & $\ldots$ & $\ldots$ & . \\
\hline & $p$ & $\mathrm{n}_{\mathrm{p} 1}$ & $\mathrm{n}_{\mathrm{p} 2}$ & $\mathrm{n}_{\mathrm{p} 3}$ & & $n_{p q}$ & $\mathrm{n}_{\mathrm{p}}$ \\
\hline Total & & $\mathrm{n}_{1}$ & $\mathrm{n} .2$ & $\mathrm{n}_{3}$ & $\ldots$ & $\mathrm{n}_{\mathrm{g}}$ & $n_{n}=n$ \\
\hline
\end{tabular}

Fonte: Mingoti (2005).

A matriz de correspondência é uma matriz $P$ de dimensões $p \times q$ construída das proporções $p_{i j}=\frac{n_{i j}}{n}$. A matriz de perfil das linhas é uma matriz diagonal denotada por $D_{r}$, cujos elementos diagonais são $r^{\prime}=\frac{n_{1}}{n} \frac{n_{2}}{n} \ldots \frac{n_{p}}{n}$. A matriz de perfil das colunas também é diagonal, denotada por $\mathrm{D}_{c^{\prime}}$, cujos elementos diagonais são iguais a $c^{\prime}=$ $\frac{n_{1}}{n} \frac{n_{2}}{n} \ldots \frac{n_{q}}{n}$. Considerando a matriz $\tilde{P}=p-r c^{\prime}$ esta matriz tem entradas do tipo, $\left(p_{i j}-\frac{n_{i}}{n} \frac{n_{j}}{n}\right)$ ou seja, como afirma Mingoti (2005, p. 259) "representam uma comparação da proporção observada no interior de cada cela da tabela, com aquela esperada sob um modelo no quais as variáveis X e Y são independentes". Com efeito, a análise de correspondências múltiplas pode ser tratada pela análise das componentes principais, decompondo-a matriz em seus autovalores e autovetores (GAMBIRAGE, et al., 2017).

As coordenadas principais das linhas da matriz $\mathrm{P}$ são dadas por $\mathrm{Y}_{\mathrm{pxq}}=$ $\mathrm{D}_{r}^{-1} \mathrm{~A}_{\mathrm{pxq}} \Lambda_{\mathrm{kxk}} \mathrm{e}$ as coordenadas principais das colunas são dadas por $\mathrm{Z}_{\mathrm{pxq}}=$ $\mathrm{D}_{r}^{-1} \mathrm{~B}_{\mathrm{pxq}} \Lambda_{\mathrm{kxk}}$. Mingoti (2005, p. 260) afirma que "as duas primeiras coordenadas principais das linhas e das colunas são as mais representativas em termos de associação" que existe entre as variáveis $\mathrm{X}$ e $\mathrm{Y}$. 


\section{DESCRIÇÃO E ANÁLISE DOS RESULTADOS}

\subsection{CARACTERIZAÇÃO DAS EMPRESAS E DOS RESPONDENTES}

Por meio da Tabela 2 observa-se algumas características das empresas pesquisadas.

Tabela 2 - Caracterização das empresas

\begin{tabular}{|c|c|c|c|}
\hline Variável & Descrição & Freq. & Perc. \\
\hline \multirow{8}{*}{$\begin{array}{c}\text { Tempo que a } \\
\text { empresa existe } \\
\text { no mercado }\end{array}$} & Até 5 anos & 18 & $24,7 \%$ \\
\hline & De 6 a 10 anos & 22 & $30,1 \%$ \\
\hline & De 11 a 15 anos & 8 & $11 \%$ \\
\hline & De 16 a 20 anos & 5 & $6,8 \%$ \\
\hline & De 21 a 25 anos & 3 & $4,1 \%$ \\
\hline & De 26 a 30 anos & 3 & $4,1 \%$ \\
\hline & Acima de 30 anos & 14 & $19,2 \%$ \\
\hline & TOTAL & 73 & $100 \%$ \\
\hline \multirow{4}{*}{$\begin{array}{l}\text { Setor da } \\
\text { empresa }\end{array}$} & Comércio & 33 & $45,2 \%$ \\
\hline & Indústria & 23 & $31,5 \%$ \\
\hline & Prestação de Serviço & 17 & $23,3 \%$ \\
\hline & TOTAL & 73 & $100 \%$ \\
\hline \multirow{8}{*}{$\begin{array}{l}\text { Número de } \\
\text { funcionários }\end{array}$} & Até 5 & 45 & $61,6 \%$ \\
\hline & De 6 a 10 & 8 & $11 \%$ \\
\hline & De 11 a 15 & 7 & $9,6 \%$ \\
\hline & De 16 a 20 & 3 & $4,1 \%$ \\
\hline & De 21 a 25 & - & - \\
\hline & De 26 a 30 & 4 & $5,5 \%$ \\
\hline & Acima de 30 & 6 & $8,2 \%$ \\
\hline & TOTAL & 73 & $100 \%$ \\
\hline
\end{tabular}

Fonte: Dados da pesquisa.

Ao analisar a tabela verifica-se que 22 empresas, o que corresponde a $30,10 \%$ da amostra estão no mercado de 6 a 10 anos, na sequência, tem-se 18 empresas $(24,7 \%)$, que estão até 5 anos no mercado. O setor de atuação que predominou foi o comércio $(45,2 \%)$, seguido da indústria $(31,5 \%)$ e da prestação de serviço $(23,3 \%)$. Quanto ao número de funcionários, $61,6 \%$ tem até 5 funcionários. Na Tabela 3 visualiza-se a caracterização dos respondentes. 
Utilização de informações contábeis e gerenciais por parte dos empresários de micros e pequenas empresas

Tabela 3 - Caracterização dos respondentes

\begin{tabular}{|c|c|c|c|}
\hline Variável & Descrição & Freq. & Perc. \\
\hline \multirow{6}{*}{ Faixa etária } & Até 25 anos & 16 & $21,90 \%$ \\
\hline & Entre 26 e 35 anos & 24 & $32,90 \%$ \\
\hline & Entre 36 e 45 anos & 12 & $16,4 \%$ \\
\hline & Entre 46 e 55 anos & 13 & $17,8 \%$ \\
\hline & Acima de 55 anos & 8 & $11 \%$ \\
\hline & TOTAL & 73 & $100 \%$ \\
\hline \multirow{9}{*}{ Escolaridade } & Ensino fundamental incompleto & 4 & $5,5 \%$ \\
\hline & Ensino fundamental & 5 & $6,8 \%$ \\
\hline & Ensino médio incompleto & 4 & $5,5 \%$ \\
\hline & Ensino médio completo & 12 & $16,4 \%$ \\
\hline & Curso Técnico Profissionalizante & 4 & $5,5 \%$ \\
\hline & Superior incompleto & 20 & $27,4 \%$ \\
\hline & Superior completo & 20 & $27,4 \%$ \\
\hline & Pós-Graduação & 4 & $5,5 \%$ \\
\hline & TOTAL & 73 & $100 \%$ \\
\hline
\end{tabular}

Fonte: Dados da pesquisa.

A maioria dos respondentes tem até 35 anos (54,80\%) e possui ensino superior incompleto ou completo $(54,80 \%)$. Visualiza-se na Tabela 4 o uso das informações contábeis e gerenciais.

Tabela 4 - Informações contábeis e gerenciais

\begin{tabular}{|c|c|c|c|}
\hline Variável & Descrição & Freq. & Perc. \\
\hline \multirow{5}{*}{$\begin{array}{l}\text { Realização de } \\
\text { cursos de gestão } \\
\text { por ano }\end{array}$} & 1 até 3 & 70 & $95,9 \%$ \\
\hline & 4 até 6 & 2 & $2,7 \%$ \\
\hline & 7 até 8 & - & - \\
\hline & Mais de 8 & 1 & $1,4 \%$ \\
\hline & TOTAL & 73 & $100 \%$ \\
\hline \multirow{11}{*}{$\begin{array}{c}\text { Relatórios } \\
\text { gerenciais } \\
\text { recebidos da } \\
\text { Contabilidade }\end{array}$} & Fluxo de Caixa & 29 & $39,7 \%$ \\
\hline & Balanço Patrimonial & 28 & $38,4 \%$ \\
\hline & DRE & 22 & $30,1 \%$ \\
\hline & Relatórios fiscais e trabalhistas & 30 & $41,1 \%$ \\
\hline & Relatório de entrada e saída de mercadoria & 16 & $21,9 \%$ \\
\hline & Faturamento & 32 & $43,8 \%$ \\
\hline & Balancete & 13 & $17,8 \%$ \\
\hline & Contas a pagar & 12 & $16,4 \%$ \\
\hline & $\begin{array}{l}\text { Informações recebidas pessoalmente do } \\
\text { contador }\end{array}$ & 21 & $28,8 \%$ \\
\hline & Nenhum & 2 & $2,7 \%$ \\
\hline & $\begin{array}{l}\text { Eu peço os balancetes, eles não mandam } \\
\text { nada }\end{array}$ & 1 & $1,4 \%$ \\
\hline \multirow{3}{*}{$\begin{array}{c}\text { Utiliza a } \\
\text { contabilidade de } \\
\text { apoio a gestão }\end{array}$} & Sim & 36 & $49,3 \%$ \\
\hline & Não & 37 & $50,7 \%$ \\
\hline & TOTAL & 73 & $100 \%$ \\
\hline \multirow{7}{*}{$\begin{array}{c}\text { Área em que a } \\
\text { informação contábil } \\
\text { é mais útil }\end{array}$} & Contábil & 15 & $20,5 \%$ \\
\hline & Controle Gerencial e Tomada de Decisão & 14 & $19,2 \%$ \\
\hline & Fiscal & 33 & $45,2 \%$ \\
\hline & Planejamento & 3 & $4,1 \%$ \\
\hline & Trabalhista & 7 & $9,6 \%$ \\
\hline & Nenhuma área & 1 & $1,4 \%$ \\
\hline & TOTAL & 73 & $100 \%$ \\
\hline
\end{tabular}

Ágora: R. Divulg. Cient., v. 23, n. 2, p. 73-94, jul./dez. 2018 (ISSNe 2237-9010) 
(Conclusão...)

\begin{tabular}{c|l|c|c}
\hline Variável & \multicolumn{1}{|c|}{ Descrição } & Freq. & Perc. \\
\hline \multirow{4}{*}{$\begin{array}{c}\text { Recursos utilizados } \\
\text { para tomada de } \\
\text { decisão }\end{array}$} & Bancos de Dados & 16 & $21,9 \%$ \\
\cline { 2 - 4 } & Relatórios Contábeis & 26 & $35,6 \%$ \\
\cline { 2 - 4 } & Sistema Gerencial & 1 & $1,4 \%$ \\
\cline { 2 - 4 } & Baseados na experiência & 37 & $50,7 \%$ \\
\cline { 2 - 4 } & Baseados na intuição & 15 & $20,2 \%$ \\
\cline { 2 - 4 } & Consultas a internet & 22 & $30,1 \%$ \\
\cline { 2 - 4 } & Relatórios Manuais & 3 & $4,1 \%$ \\
\cline { 2 - 4 } & Nenhum & 24 & $32,9 \%$ \\
\cline { 2 - 4 } & Pesquisa de Mercado & 14 & $19,2 \%$ \\
\cline { 2 - 4 } & Planilhas estatísticas & & $(2 \%$ \\
\hline
\end{tabular}

Fonte: Dados da pesquisa.

São realizados de 1 até 3 cursos de gestão por ano, resposta dada por 95,9\% dos respondentes. Relatórios sobre o faturamento, fiscais e trabalhistas, fluxo de caixa, balanço patrimonial foram os respondidos com maior frequência no que se refere aos relatórios gerenciais recebidos da contabilidade. Aproximadamente metade $50,7 \%$ dos respondentes não utiliza a contabilidade de apoio a gestão.

A maioria dos respondentes declara que a informação contábil é mais útil na área fiscal $(45,2 \%)$, já em relação ao aspecto contábil $(20,5 \%)$, controle gerencial e tomada de decisão $(19,2 \%)$, os percentuais foram menores. A maioria das tomadas de decisão é baseada na experiência $(50,7 \%)$, nos relatórios contábeis $(35,6 \%)$, pesquisa de mercado $(32,9 \%)$ e relatórios manuais $(30,1 \%)$. Por meio da Tabela 5 apresenta-se as informações de gerenciamento e sistema de controle.

Tabela 5 - Informações de gerenciamento e sistema de controle

\begin{tabular}{|c|c|c|c|}
\hline Variável & Descrição & Freq. & Perc. \\
\hline \multirow{19}{*}{$\begin{array}{c}\text { Gerenciamento } \\
\text { e Sistema de } \\
\text { Controle }\end{array}$} & Objetivos Estratégicos & 14 & $19,2 \%$ \\
\hline & Gerenciamento de Fluxo de Caixa & 29 & $39,7 \%$ \\
\hline & Previsões de Fluxo de Caixa & 20 & $27,4 \%$ \\
\hline & Controles de Compras & 42 & $57,5 \%$ \\
\hline & Controles de pagamento de credores & 33 & $45,2 \%$ \\
\hline & Controle de estoque & 44 & $60,3 \%$ \\
\hline & Controle de devedores & 30 & $41,1 \%$ \\
\hline & Gestão de financiamento & 13 & $17,8 \%$ \\
\hline & Margem e gerenciamento de custos & 11 & $15,1 \%$ \\
\hline & Folhas de tempo de trabalho e custos de... & 14 & $19,2 \%$ \\
\hline & Preços e Markup & 8 & $11 \%$ \\
\hline & Controle de Margem Bruta & 13 & $17,8 \%$ \\
\hline & Análise de Equilíbrio & 6 & $8,2 \%$ \\
\hline & Sensibilidade de custo $\mathrm{x}$ volume $\mathrm{x}$ lucro & 3 & $4,1 \%$ \\
\hline & Orçamento básico & 18 & $24,7 \%$ \\
\hline & Contas de lucros e perdas & 18 & $24,7 \%$ \\
\hline & Balanço Patrimonial & 17 & $23,3 \%$ \\
\hline & Demonstração do Resultado do Exercício & 14 & $19,2 \%$ \\
\hline & Monitoramento da satisfação do cliente & 17 & $23,3 \%$ \\
\hline
\end{tabular}

Fonte: Dados da pesquisa.

Ágora: R. Divulg. Cient., v. 23, n. 2, p. 73-94, jul./dez. 2018 (ISSNe 2237-9010) 
Controle de estoque $(60,3 \%)$, controle de compras $(57,5 \%)$, controles de pagamento de credores $(45,2 \%)$, controle de devedores $(41,1 \%)$ e gerenciamento de fluxo de caixa $(39,7 \%)$ são as informações de gerenciamento e sistema de controle respondidos pela maioria. Nos quesitos controle de estoque, contas a receber e controle de compras o resultado está em consonância com o estudo de Ueno e Casa Nova (2006); em relação ao controle de pagamento confirma o achado de Caneca (2008). Na Tabela 6 apresenta-se as competências gerenciais no que se refere aos aspectos sociais.

Tabela 6 - Competências gerenciais - Sociais

\begin{tabular}{|c|c|c|c|c|c|c|c|c|c|c|}
\hline \multirow[t]{2}{*}{ Descrição } & \multicolumn{2}{|c|}{ 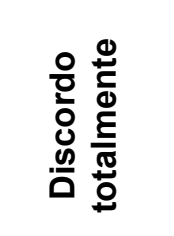 } & \multicolumn{2}{|r|}{$\begin{array}{l}0 \\
\frac{0}{0} \\
0 \\
\frac{0}{0} \\
\frac{0}{0}\end{array}$} & \multicolumn{2}{|c|}{ 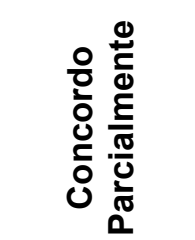 } & \multicolumn{2}{|r|}{$\begin{array}{l}\circ \\
0 \\
0 \\
0 \\
0 \\
0\end{array}$} & \multicolumn{2}{|c|}{ 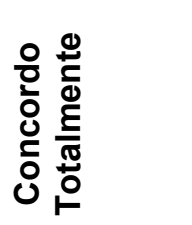 } \\
\hline & F. & $\%$ & F. & $\%$ & F. & $\%$ & F. & $\%$ & F. & $\%$ \\
\hline $\begin{array}{l}\text { Suporto pressões e adapto-me } \\
\text { a situações e contextos } \\
\text { variados }\end{array}$ & 3 & $4,1 \%$ & 6 & $8,2 \%$ & 32 & $43,8 \%$ & 24 & $32,9 \%$ & 8 & $11 \%$ \\
\hline $\begin{array}{l}\text { Invisto no fortalecimento da } \\
\text { equipe }\end{array}$ & - & - & 4 & $5,5 \%$ & 23 & $31,5 \%$ & 30 & $41,1 \%$ & 16 & $21,9 \%$ \\
\hline $\begin{array}{l}\text { Torno claro para a equipe os } \\
\text { objetivos a serem atingidos }\end{array}$ & - & - & 4 & $5,5 \%$ & 22 & $30,1 \%$ & 31 & $42,5 \%$ & 16 & $21,9 \%$ \\
\hline $\begin{array}{l}\text { Adapto a linguagem à situação } \\
\text { e ao ouvinte }\end{array}$ & - & - & 2 & $2,7 \%$ & 25 & $34,2 \%$ & 28 & $38,4 \%$ & 18 & $24,7 \%$ \\
\hline $\begin{array}{l}\text { Ouço os funcionários com } \\
\text { atenção }\end{array}$ & - & - & 1 & $1,4 \%$ & 21 & $28,8 \%$ & 34 & $46,6 \%$ & 17 & $23,3 \%$ \\
\hline $\begin{array}{l}\text { Mantenho as pessoas } \\
\text { informadas sobre os planos e } \\
\text { processos }\end{array}$ & 1 & $1,4 \%$ & 7 & $9,6 \%$ & 16 & $21,9 \%$ & 35 & $47,9 \%$ & 14 & $19,2 \%$ \\
\hline $\begin{array}{l}\text { Comunico-me de modo a gerar } \\
\text { entusiasmo }\end{array}$ & - & - & 4 & $5,5 \%$ & 20 & $27,4 \%$ & 39 & $53,4 \%$ & 10 & $13,7 \%$ \\
\hline $\begin{array}{l}\text { Apresento-me positivamente } \\
\text { (estado espírito) }\end{array}$ & 1 & $1,4 \%$ & 3 & $4,1 \%$ & 21 & $28,8 \%$ & 29 & $39,7 \%$ & 19 & $26 \%$ \\
\hline $\begin{array}{l}\text { Desenvolvo relacionamentos } \\
\text { com clientes e colegas }\end{array}$ & - & - & 3 & $4,1 \%$ & 19 & $26 \%$ & 24 & $32,9 \%$ & 27 & $37 \%$ \\
\hline $\begin{array}{l}\text { Utilizo métodos para conduzir } \\
\text { reuniões de trabalho }\end{array}$ & 8 & $11 \%$ & 14 & $19,2 \%$ & 23 & $31,5 \%$ & 21 & $28,8 \%$ & 7 & $9,6 \%$ \\
\hline
\end{tabular}

Fonte: Dados da pesquisa.

As competências gerenciais relacionadas aos aspectos sociais que tiveram maior número de respostas foram: desenvolvo relacionamentos com clientes e colegas (37\% concorda totalmente), comunico-me de modo a gerar entusiasmo (53,4\% concorda) e suporto pressões e adapto-me a situações e contextos variados (43,8\% concorda parcialmente). Denotando-se assim que os gestores possuem 
entusiasmo na condução do negócio, conseguem lida com situações de pressão e estresse e também possuem certa capacidade de manter bom relacionamento com clientes e colegas. Na Tabela 7 visualiza-se as competências técnico profissionais.

Tabela 7 - Competências gerenciais - Competências Técnico Profissionais

\begin{tabular}{|c|c|c|c|c|c|c|c|c|c|c|}
\hline \multirow[t]{2}{*}{ Descrição } & \multicolumn{2}{|c|}{ 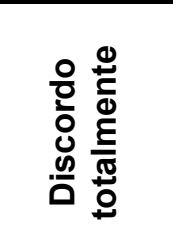 } & \multicolumn{2}{|r|}{ 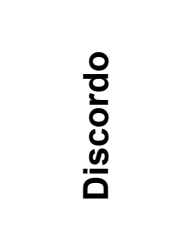 } & \multicolumn{2}{|c|}{ 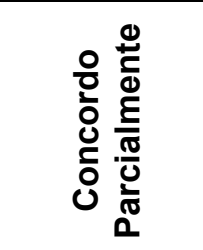 } & \multicolumn{2}{|r|}{$\begin{array}{l}0 \\
0 \\
0 \\
0 \\
0 \\
0\end{array}$} & \multicolumn{2}{|c|}{ 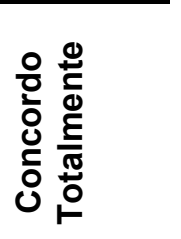 } \\
\hline & F. & $\%$ & F. & $\%$ & F. & $\%$ & F. & $\%$ & F. & $\%$ \\
\hline $\begin{array}{lcc}\text { Utilizo técnicas } & \text { de } \\
\text { planejamento estratégico } & \end{array}$ & 3 & $4,1 \%$ & 8 & $11 \%$ & 21 & $28,8 \%$ & 29 & $39,7 \%$ & 12 & $16,4 \%$ \\
\hline $\begin{array}{lll}\text { Monitoro a qualidade } & \text { e } & \text { o } \\
\text { andamento dos serviços }\end{array}$ & - & - & 5 & $6,8 \%$ & 11 & $15,1 \%$ & 33 & $45,2 \%$ & 24 & $32,9 \%$ \\
\hline $\begin{array}{lll}\begin{array}{l}\text { Conheço } \\
\text { negociação }\end{array} & \text { técnicas } & \text { de } \\
\end{array}$ & 2 & $2,7 \%$ & 3 & $4,1 \%$ & 29 & $39,7 \%$ & 24 & $32,9 \%$ & 14 & $20,5 \%$ \\
\hline $\begin{array}{l}\text { Faço escolhas a partir do auxílio } \\
\text { de funcionários }\end{array}$ & 1 & $1,4 \%$ & 9 & $12,3 \%$ & 23 & $31,5 \%$ & 28 & $38,4 \%$ & 12 & $16,4 \%$ \\
\hline $\begin{array}{l}\text { Gerencio conflitos internos e } \\
\text { externos }\end{array}$ & - & - & 7 & $9,6 \%$ & 21 & $28,8 \%$ & 31 & $42,5 \%$ & 14 & $19,2 \%$ \\
\hline Delego atividades de trabalho & - & - & 1 & $1,4 \%$ & 25 & $34,2 \%$ & 24 & $32,9 \%$ & 23 & $31,5 \%$ \\
\hline $\begin{array}{l}\text { Conheço os produtos e serviços } \\
\text { oferecidos na empresa }\end{array}$ & - & - & 4 & $5,5 \%$ & 9 & $12,3 \%$ & 24 & $32,9 \%$ & 36 & $49,3 \%$ \\
\hline $\begin{array}{lr}\begin{array}{l}\text { Acompanho } \\
\text { rotinas } \\
\text { operacionais }\end{array} & \begin{array}{r}\text { pessoalmente } \\
\text { processos }\end{array} \\
\end{array}$ & - & - & 7 & $9,6 \%$ & 14 & $19,2 \%$ & 23 & $31,5 \%$ & 29 & $39,7 \%$ \\
\hline $\begin{array}{l}\text { Utilizo métodos para conduzir } \\
\text { reuniões de trabalho }\end{array}$ & 8 & $11 \%$ & 14 & $19,2 \%$ & 23 & $31,5 \%$ & 21 & $28,8 \%$ & 7 & $9,6 \%$ \\
\hline
\end{tabular}

Fonte: Dados da pesquisa.

Quanto as competências técnico profissionais, 49,3\% concorda totalmente com a afirmação de que conhece os produtos e serviços oferecidos pela empresa, $45,2 \%$ concorda com a assertiva de que monitora a qualidade e o andamento dos serviços, e $39,7 \%$ concorda parcialmente com a afirmativa de que conhece técnicas de negociação. Desta forma observa-se que as competências técnico profissionais que se destacam nos gestores são o conhecimento dos seus produtos e serviços, capacidade de monitorar a qualidade e a rotina de trabalho e possui certo conhecimento de técnicas de negociação. 
Tabela 8 - Competências gerenciais - Competências de Negócios

\begin{tabular}{|c|c|c|c|c|c|c|c|c|c|c|}
\hline \multirow[t]{2}{*}{ Descrição } & \multicolumn{2}{|c|}{ 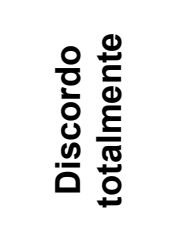 } & \multicolumn{2}{|r|}{ 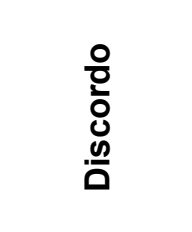 } & \multicolumn{2}{|c|}{ 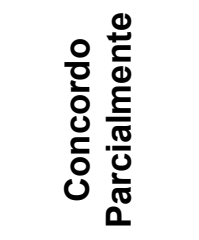 } & \multicolumn{2}{|r|}{$\begin{array}{l}\text { 은 } \\
\text { 0 } \\
\text { 잉 }\end{array}$} & \multicolumn{2}{|c|}{ 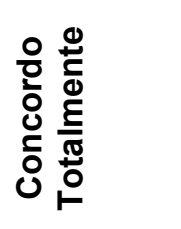 } \\
\hline & F. & $\%$ & F. & $\%$ & F. & $\%$ & F. & $\%$ & F. & $\%$ \\
\hline $\begin{array}{l}\text { Compreendo como a empresa } \\
\text { interage com os clientes }\end{array}$ & 1 & $1,4 \%$ & 3 & $4,1 \%$ & 18 & $24,7 \%$ & 34 & $46,6 \%$ & 17 & $23,3 \%$ \\
\hline $\begin{array}{lr}\text { Mantenho-me atento } \\
\text { oportunidades negociais }\end{array}$ & - & - & 7 & $9,6 \%$ & 14 & $19,2 \%$ & 32 & $43,8 \%$ & 20 & $27,4 \%$ \\
\hline $\begin{array}{l}\text { Foco atenção nos resultados } \\
\text { financeiros }\end{array}$ & 1 & $1,4 \%$ & 4 & $5,5 \%$ & 9 & $12,3 \%$ & 31 & $42,5 \%$ & 28 & $38,4 \%$ \\
\hline $\begin{array}{l}\text { Desenvolvo uma rede } \\
\text { informações estratégicas }\end{array}$ & 1 & $1,4 \%$ & 10 & $13,7 \%$ & 27 & $37 \%$ & 29 & $39,7 \%$ & 6 & $8,2 \%$ \\
\hline $\begin{array}{l}\text { Procuro informações em várias } \\
\text { fontes }\end{array}$ & 1 & $1,4 \%$ & 5 & $6,8 \%$ & 22 & $30,1 \%$ & 30 & $41,1 \%$ & 15 & $20,5 \%$ \\
\hline $\begin{array}{l}\text { Oriento as ações pelas } \\
\text { necessidades dos clientes }\end{array}$ & 1 & $1,4 \%$ & 3 & $4,1 \%$ & 13 & $17,8 \%$ & 42 & $57,5 \%$ & 14 & $19,2 \%$ \\
\hline $\begin{array}{l}\text { Estabeleço parcerias com os } \\
\text { clientes }\end{array}$ & - & - & 5 & $6,8 \%$ & 16 & $21,9 \%$ & 31 & $42,5 \%$ & 21 & $28,8 \%$ \\
\hline $\begin{array}{l}\text { Sou participativo em eventos na } \\
\text { comunidade (cidade) }\end{array}$ & 4 & $5,5 \%$ & 12 & $16,4 \%$ & 26 & $35,6 \%$ & 20 & $27,4 \%$ & 11 & $15,1 \%$ \\
\hline $\begin{array}{l}\text { Pesquiso sobre os costumes e } \\
\text { tradições que afetam os clientes }\end{array}$ & 2 & $2,7 \%$ & 13 & $17,8 \%$ & 21 & $28,8 \%$ & 26 & $35,6 \%$ & 11 & $15,1 \%$ \\
\hline
\end{tabular}

Fonte: Dados da pesquisa.

No que tange as competências de negócios, focar a atenção nos resultados financeiros teve a maior frequência de respostas com concordância total, sendo respondido por $38,4 \%$, confirmando assim a utilização da contabilidade apenas com o objetivo de controle.

Após a estatística descritiva, o uso da Anacor permitiu verificar as características das empresas e dos respondentes que usam e não usam as informações contábeis de apoio a gestão. Por meio da Figura 1, apresenta-se as informações obtidas com a aplicação da Anacor. 
Figura 1 - Características das empresas que USAM e NÃO USAM as informações contábeis e gerenciais

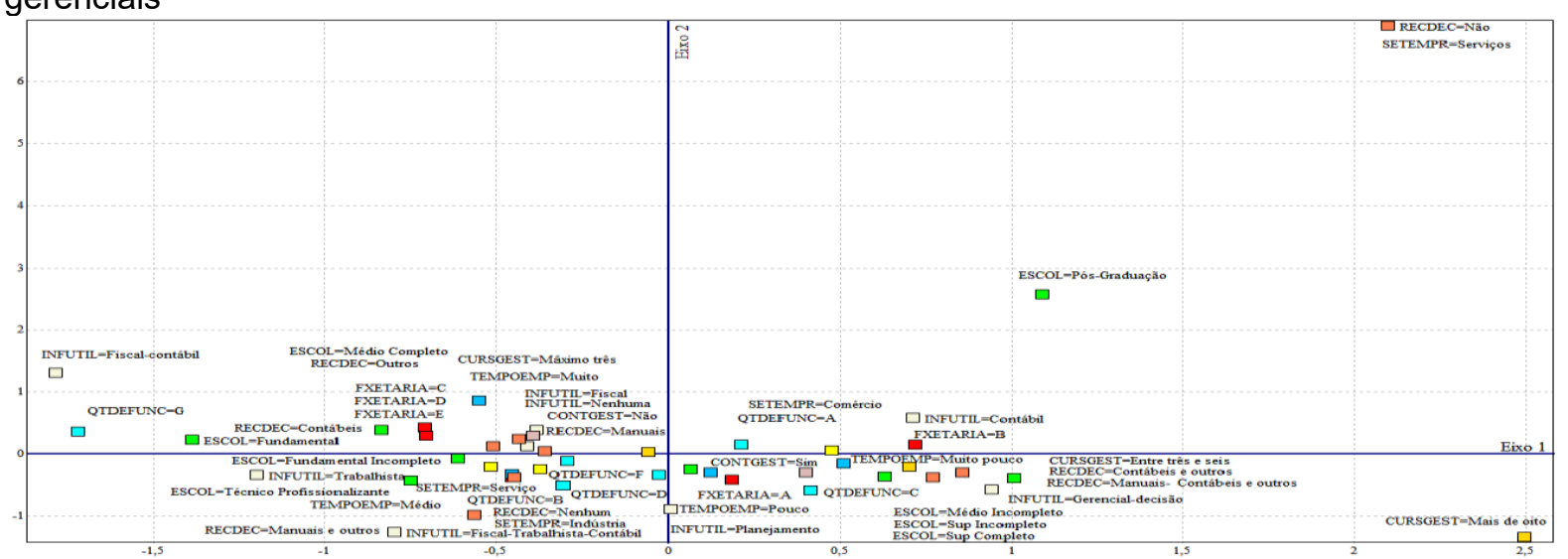

Legenda: FXETARIA (faixa etária) = A (até 25 anos); B ( 26 a 35 anos); C ( 36 a 45 anos); D (46 a 55 anos); E (55 ou mais). ESCOL (escolaridade). TEMPOEMP (tempo de empresa) = pouco (até 15); médio (de 16 a 25); muito (de 26 a mais do 30) SETEMPR (setor da empresa); QTDEFUNC (quantidade de funcionários) = A (até 5); B (De 6 a 10); C (De 11 a 15); D (De 16 a 10); E (De 21 a 25); F (De 26 a 30); G (Acima de 30). CURSGEST (quantidade de curso de gestão por ano). INFUTIL (área em que a informação contábil é mais útil). CONTGEST (uso da contabilidade para gestão). RECDEC (recursos utilizados para tomada de decisão).

Fonte: Dados da pesquisa.

A Figura 1 evidencia que as empresas que não utilizam a contabilidade para apoio a gestão possuem as seguintes características: ensino fundamental, ensino médio e técnico profissionalizante como escolaridade (ESCOL), gestores com faixa etária (FXETARIA) de 36 a 55 anos, empresas com tempo de existência (TEMPEMPR) de 16 a mais de 30 anos, prestação de serviço e indústria como setor da empresa (SETEMPR), realizam no máximo três cursos de gestão por ano (CURSGEST), a informação contábil é mais útil (INFUTIL) para a área fiscal, contábil, trabalhista e nenhuma utilidade, os recursos utilizados para a tomada de decisão (RECDEC) são relatórios manuais e contábeis.

As empresas que utilizam a contabilidade para apoio a gestão possuem as seguintes características: ensino médio, superior incompleto e completo como escolaridade (ESCOL), gestores com faixa etária (FXETARIA) de até 35 anos, empresas com tempo de existência (TEMPEMPR) de até 15 anos, comércio como setor da empresa (SETEMPR), realizam entre três a seis cursos de gestão por ano (CURSGEST), a informação contábil é mais útil (INFUTIL) para planejamento, gerencial-decisão, os recursos utilizados para a tomada de decisão (RECDEC) são relatórios manuais, contábeis e outros.

Verifica-se que a escolaridade em nível de Pós-graduação apresentou uma certa distância do uso ou não da contabilidade para apoio a gestão, o que permite 
inferir que esta não possui relação. Essa constatação pode ser explicada pelo fato de que o ensino superior já é suficiente para destacar a relevância da contabilidade.

Pode-se inferir com base nos resultados que, as empresas com menor tempo de mercado e com gestores que possuem educação continuada tendem a utilizar a contabilidade para fins gerenciais, enquanto que empresas com maior tempo de mercado e com pouca educação continuada, tendem a utilizar a contabilidade somente para atender exigências fiscais.

\section{CONSIDERAÇÕES FINAIS}

A presente pesquisa consistia em analisar as informações contábeis e gerenciais utilizadas pelos gestores de micros e pequenas empresas, tratando-se de um estudo descritivo, de levantamento e quantitativo.

A amostra pesquisada foi composta por 73 micros e pequenas empresas do Vale do Itajaí/SC, sendo semelhante aos estudos de Kos et al. (2014) que investigou 50 micros e pequenas paranaenses e de Santos et al. (2016) que utilizou 41 micros e pequenas empresas para compor a sua amostra.

A maioria das micros e pequenas empresas investigadas é está no mercado de 6 a 10 anos, atuam no comércio e possuem até cinco funcionários. Quanto aos respondentes, a maioria tem até 35 anos e possui ensino superior incompleto ou completo.

Quanto as informações contábeis e gerenciais, os resultados demonstram que a maioria dos gestores das micros e pequenas empresas consideram que contabilidade é realizada primordialmente para fins fiscais.

Em relação as informações de gerenciamento e sistemas de controle, constatou-se que os relatórios sobre o faturamento, fiscais e trabalhistas são os mais utilizados e aproximadamente metade dos respondentes não utiliza a contabilidade para apoio a gestão, sendo que a tomada de decisão é baseada principalmente na experiência do gestor. Controle de estoque, controle de compras, controles de pagamento de credores, controle de devedores e gerenciamento de fluxo de caixa são as informações de gerenciamento e sistema de controle respondidos pela maioria.

Esses achados são semelhantes aos encontrados por Moreira et al., Kos et al. (2014), Lucena, Vasconcelos e Marcelino (2015) e Santos et al. (2016). 
No tocante as competências gerenciais os resultados apontaram que as habilidades relacionadas aos aspectos sociais que tiveram maior número de respostas, foram: a capacidade de ouvir os funcionários com atenção, de adaptar a linguagem à situação e ao ouvinte e de desenvolver relacionamentos com os clientes e colegas. Quanto as competências técnico profissionais observou que a maioria possui a habilidade de delegar atividaconhece os produtos e serviços oferecidos na empresa e monitora a qualidade e o andamento dos serviços.

Diante dos dados apresentados, segregou-se por meio da ANACOR ASs características das micros e pequenas empresas que utilizam a contabilidade para apoio a gestão, inferindo-se que, as empresas com menor tempo de mercado e com gestores que possuem educação continuada tendem a utilizar a contabilidade para fins gerenciais, enquanto que empresas com maior tempo de mercado e com pouca educação continuada, tendem a utilizar a contabilidade somente para fins fiscais.

\section{REFERÊNCIAS}

ABOR, Joshua; QUARTEY, Peter. Issues in SME development in Ghana and South Africa. International Research Journal of Finance and Economics, v. 39, n. 6, p. 215-228, 2010.

AHMAD, Kamilah; MOHAMED ZABRI, Shafie. Factors explaining the use of management accounting practices in Malaysian medium-sized firms. Journal of Small Business and Enterprise Development, v. 22, n. 4, p. 762-781, 2015.

ALBUQUERQUE, Alexandre Farias. Gestão estratégica das informações internas na pequena empresa: estudo comparativo de casos em empresas do setor de serviços (hoteleiro) da região de Brotas-SP. 2004. Tese (Doutorado) Universidade de São Paulo. São Paulo, 2004.

ALONSO-ALMEIDA, María del Mar et al. Sustainability in small tourist businesses: the link between initiatives and performance. Current Issues in Tourism, v. 21, n. 1, p. 1-20, 2018.

BARBOZA, Ricardo Augusto Bonotto; FONSECA, Sergio Azevedo; FREITAS RAMALHEIRO, Geralda Cristina de. O papel das políticas públicas para potencializar a inovação em pequenas empresas de base tradicional. REGE-Revista de Gestão, v. 24, n. 1, p. 58-71, 2017.

BATY, Gordon B. Pequenas e médias empresas dos anos 90: guia do consultor e do empreendedor. Rio de Janeiro: Makron Books, 1994. 
BEATTY, Anne; LIAO, Scott. Financial accounting in the banking industry: A review of the empirical literature. Journal of Accounting and Economics, v. 58, n.2-3, p. 339-383, 2014.

BERNARDES, Danulia Peixer Galm; MIRANDA, Luis Carlos. Quatro Historias Da Utilização De Informação Econômico-Financeira Nas Micro E Pequenas Empresas: Lições Para Futuros Empreendedores. Revista da Micro e Pequena Empresa, v. 5, n. 3, p. 84-98, 2011.

BORGES, Lara Fabiana Morais; LEAL, Edvalda Araújo. Contabilidade gerencial: a utilização das informações contábeis gerenciais pelos gestores das micro e pequenas empresas. SEGET, SIMPÓSIO DE EXCELÊNCIA EM GESTÃO E TECNOLOGIA, v. 12, 2012.

BRASIL. Lei Complementar $n^{\circ}$ 123, de 14 de dezembro de 2006. Institui o Estatuto Nacional da Microempresa e da Empresa de Pequeno Porte; altera dispositivos das Leis nㅇ.212 e 8.213, ambas de 24 de julho de 1991, da Consolidação das Leis do Trabalho - CLT, aprovada pelo Decreto-Lei no 5.452, de 1o de maio de 1943, da Lei no 10.189 , de 14 de fevereiro de 2001, da Lei Complementar no 63, de 11 de janeiro de 1990; e revoga as Leis no 9.317, de 5 de dezembro de 1996, e 9.841, de 5 de outubro de 1999. Diário Oficial da União, Brasília, DF, 06 mar. 2012.Disponível em <http://www.planalto.gov.br/ccivil_03/leis/lcp/lcp123.htm >. Acesso em: 17 ago. 2018.

CANECA, Roberta Lira. Oferta e Procura de Serviços Contábeis para Micro, Pequenas e Médias Empresas: um estudo comparativo das percepções dos empresários e contadores. 2008.

CANECA, Roberta Lira et al. A influência da oferta de contabilidade gerencial na percepção da qualidade dos serviços contábeis prestados aos gestores de micro, pequenas e médias empresas. Pensar contábil, v. 11, n. 43, 2009.

COLLINS, Daniel W.; MAYDEW, Edward L.; WEISS, Ira S. Mudanças na relevância do valor dos lucros e valores contábeis nos últimos quarenta anos. Jornal de contabilidade e economia, v. 24, n. 1, p. 39-67, 1997.

CONSELHO FEDERAL DE CONTABILIDADE. Resolução 1374/11. Dá nova redação à NBC TG ESTRUTURA CONCEITUAL. Estrutura Conceitual para Elaboração e Divulgação de Relatório Contábil-Financeiro. Brasília, DF, 8 dez. 2011. Disponível em <http://www1.cfc.org.br/sisweb/SRE/docs/Res_1374.pdf> Acesso em: 15 ago. 2018.

COSTA, Rui; COSTA, Carlos. Importância das empresas de pequena e micro dimensão em termos de investimento e criação de emprego no setor do turismo no período de 1994-2006. Revista Turismo \& Desenvolvimento, n. 19, p. 85-98, 2018.

COWLING, Marc; LIU, Weixi; LEDGER, Andrew. Small business financing in the UK before and during the current financial crisis. International Small Business Journal, v. 30, n. 7, p. $778-800,2012$. 
DAI, Na; IVANOV, Vladimir; COLE, Rebel A. Entrepreneurial optimism, credit availability, and cost of financing: Evidence from US small businesses. Journal of Corporate Finance, v. 44, p. 289-307, 2017.

FÁVERO, L. P. et al. Análise de dados: Modelagem multivariada para tomada de decisões. Rio de Janeiro: Elsevier, 2009.

FATOKI, Olawale. An investigation into the financial management practices of new micro-enterprises in South Africa. Journal of social sciences, v. 33, n. 2, p. 179188, 2012.

FERNANDES, Francisco Carlos; KLANN, Roberto Carlos; FIGUEREDO, Marcelo Salmeron. A utilidade da informação contábil para a tomada de decisões: uma pesquisa com gestores alunos. Contabilidade Vista \& Revista, v. 22, n. 3, p. 99126, 2011.

FIEK, Nilton; LOOSE, Cleberson Eller. Uso das informações contábeis nas micro e pequenas empresas. Revista de Administração de Roraima-RARR, v. 7, n. 2, p. 348-365, 2018.

FRANCIS, Jennifer; SCHIPPER, Katherine. Have financial statements lost their relevance?. Journal of accounting Research, v. 37, n. 2, p. 319-352, 1999.

GAMBIRAGE, Cinara et al. Estilos de liderança predominantes em uma indústria madeireira no Meio-Oeste catarinense: um estudo de caso. Anais do VIII Colóquio de Redes, Estratégia e Inovação, 2017. Disponível em:

<http://www.nucleoestudo.ufla.br/gerei/wp-content/uploads/2018/02/ESTILOS-DELIDERAN\%C3\%87A-PREDOMINANTES-EM-UMA-IND\%C3\%9ASTRIAMADEIREIRA-NO-MEIO-OESTE-CATARINENSE.pdf>. Acesso em: 09 out. 2018.

GUPTA, Prashant; SEETHARAMAN, Arumugam; RAJ, John Rudolph. The usage and adoption of cloud computing by small and medium businesses. International Journal of Information Management, v. 33, n. 5, p. 861-874, 2013.

HALL, Matthew. Accounting information and managerial work. Accounting, Organizations and Society, v. 35, n. 3, p. 301-315, 2010.

HALL, Rosemar José et al. Contabilidade como uma ferramenta da gestão: um estudo em micro e pequenas empresas do ramo de comércio de DouradosMS/Accounting as a tool of management: a study in micro and small industry the trade Dourados-MS. Revista da Micro e Pequena Empresa, v. 6, n. 3, p. 4, 2012.

HOTHO, Sabine; CHAMPION, Katherine. Small businesses in the new creative industries: innovation as a people management challenge. Management Decision, v. 49 , n. 1, p. 29-54, 2011.

KOS, Sonia Raifur et al. Compreensão e utilização da informação contábil pelos micro e pequenos empreendedores em seu processo de gestão. Enfoque: Reflexão Contábil, v. 33, n. 3, 2014. 
LEONE, Nilda Maria de Clodoaldo Pinto et al. A dimensão física das pequenas e médias empresas (PME'S): à procura de um critério homogeneizador. Revista de Administração de Empresas, v. 31, n. 2, p. 53-59, 1991.

LUCENA, Wenner G. L. Uma contribuição ao estudo das informações contábeis geradas pelas micro e pequenas empresas localizadas na cidade de Toritama no agreste pernambucano. Dissertação (Mestrado em Ciências Contábeis) Programa Multiinstitucional e Inter-Regional de Pós-Graduação em Ciências Contábeis da UnB/UFPE/UFPB/UFRN, Brasília, 2004.

LUCENA, Wenner G. L.; VASCONCELOS, Marco Tullio de Castro; MARCELINO, Gileno Fernandes. A evidenciação das informações contábeis geradas pelas micro e pequenas empresas no processo decisório: Um estudo no setor de confecções.

Revista Reunir, v. 1, n. 1, p. 35-51, 2015.

MANO, Yukichi et al. How can micro and small enterprises in sub-Saharan Africa become more productive? The impacts of experimental basic managerial training. World Development, v. 40, n. 3, p. 458-468, 2012.

MINGOTI, S. A. Análise de dados através de métodos de estatística multivariada: uma abordagem aplicada. Belo Horizonte: UFMG, 2005.

MOHAMED ZABRI, Shafie. The determinants of capital structure among SMEs in Malaysia. 2012.

MOREIRA, Rafael de Lacerda et al. A importância da informação contábil no processo de tomada de decisão nas micro e pequenas empresas. Revista Contemporânea de Contabilidade, v. 10, n. 19, p. 119-140, 2013.

MUNGARAY LAGARDA, Alejandro; RAMÍREZ URQUIDY, Martín. Capital humano y productividad en microempresas. Investigación económica, v. 66, n. 260, p. 81115, 2007.

NAQVI, Syed Wajahat Hussain. Critical success and failure factors of entrepreneurial organizations: Study of SMEs in Bahawalpur. Journal of Public Administration and Governance, v. 1, n. 2, p. 17-23, 2011.

OLEIRO, Walter Nunes; DAMEDA, André das Neves; VICTOR, Fernanda Gomes. O uso da informação contábil na gestão de micro e pequenas empresas atendidas pelo programa de extensão empresarial NEE/FURG. SINERGIA: Revista do Instituto de Ciências Econômicas, Administrativas e Contábeis, v. 11, n. 1, p. 37-47, 2007.

OLIVEIRA CAMELO, Augusto César; MARTINS CAMELO, Érika Patrícia de S.; PAULINO, Tatiana Cristina de Brito. A importância do gerenciamento contábil para as organizações do terceiro setor. Enfoque: Reflexão Contábil, v. 25, n. 2, 2006.

PERERA, R. A. A. S.; THRIKAWALA, S. S. An empirical study of the relevance of accounting information on investor's decisions. 2010. 
PEREIRA, Frederico Cesar Mafra et al. Competitive Intelligence as a Support to the Business Strategy in Micro and Small Companies: A Study in The Aerotropolis of Belo Horizonte. Revista Ibero-Americana de Estratégia, v. 17, n. 1, p. 93, 2018.

PETRUS, Henry George et al. An investigation into causes of success and failure in small businesses within the Department of Social Development in the Eastern Cape. 2009.

RAMÍREZ ANGULO, Natanael et al. Economías de escala y rendimientos crecientes: una aplicación en microempresas mexicanas. Economía mexicana. Nueva época, v. 19, n. 2, p. 213-230, 2010.

SANTOS, Vanderlei dos; DOROW, Diego Roberto; BEUREN, Ilse Maria. Práticas gerenciais de micro e pequenas empresas. Revista Ambiente Contábil:

Universidade Federal do Rio Grande do Norte, v. 8, n. 1, p. 153-186, 2016.

SANTOS, Vanderlei dos et al. características das empresas e dos gestores que influenciam no uso de controles gerenciais. Advances in Scientific and Applied Accounting, v. 11, n. 1, p. 003-023, 2018.

SANTOS, Vanderlei dos et al. Instrumentos da contabilidade gerencial utilizados pelas micro, pequenas e médias empresas: estudo em uma prestadora de serviços contábeis e seus respectivos clientes. In: CONGRESSO BRASILEIRO DE CUSTOS. Anais do Congresso Brasileiro de Custos-ABC. 2014.

SEBRAE. Entenda o motivo do sucesso e do fracasso das empresas. Disponível em: <http://www.sebrae.com.br/sites/PortalSebrae/ufs/sp/bis/entenda-o-motivo-dosucesso-e-do-fracasso-das-empresas,b1d31ebfe6f5f510VgnVCM1000004c00210 aRCRD?codUf=26\&origem=estadual>. Acesso em: 19 ago. 2018.

Anuário do trabalho na micro e pequena empresa 2013. Disponível em <www.sebrae.com.br/Sebrae/Portal\%20Sebrae/Anexos/Anuario\%20do\%20Trabalho $\% 20 \mathrm{Na} \% 20 \mathrm{Micro} \% 20 \mathrm{e} \% 20 \mathrm{Pequena} \% 20$ Empresa_2013.pdf>. Acesso em: 19 ago. 2018.

Sobrevivência das empresas no Brasil. Disponível em

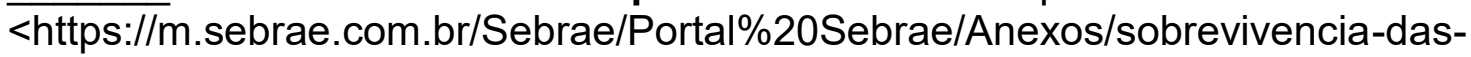
empresas-no-brasil-102016.pdf> Acesso em 19 agos. 2018.

SEMLER, Ricardo Frank. Virando a própria mesa. São Paulo: Best Seller, 1988.

SAES, Maria Sylvia Macchione; HOURNEAUX JUNIOR, Flavio. RAUSP Management Journal: changes and challenges for 2018. RAUSP Management Journal, v. 53, n. 1, p. 1-2, 2018. 
. Fatores condicionantes e taxas de sobrevivência e mortalidade das micro e pequenas empresas no Brasil 2003-2005. Brasília: Sebrae, 2007. Disponível em <http://www.bibliotecas.sebrae.com.br/chronus/ARQUIVOS CHRONUS/bds/bds.nsf/8F5BDE79736CB99483257447006CBAD3/\$File/NT0003793 6.pdf> Acesso em 09 out. 2018.

SHELDON, Dan. Recognizing failure factors helps small-business turnarounds. National Productivity Review, v. 13, n. 4, p. 533-541, 1994.

SHIBIA, Adan Guyo; BARAKO, Dulacha Galgallo. Determinants of micro and small enterprises growth in Kenya. Journal of Small Business and Enterprise Development, v. 24, n. 1, p. 105-118, 2017.

SPRINKLE, Geoffrey B. Perspectives on experimental research in managerial accounting. Accounting, Organizations and Society, v. 28, n. 2-3, p. 287-318, 2003.

STROEHER, Angela Maria. Identificação das caraterísticas das informações contábeis e sua utilização para tomada de decisão organizacional de pequenas empresas. 2005. Dissertação (Mestrado em Administração) - Universidade Federal do Rio Grande do Sul. Porto Alegre, 2005.

TUNDUI, Charles Stephen; TUNDUI, H. Microcredit, micro enterprising and repayment Myth: the case of micro and small women business entrepreneurs in Tanzania. American Journal of Business and Management, v. 2, n. 1, p. 20-30, 2013.

TUNG, Rosalie L.; AYCAN, Zeynep. Key success factors and indigenous management practices in SMEs in emerging economies. Journal of World Business, 2008.

UENO, Rodrigo Bergame; CASA NOVA, Silvia Pereira de Castro. Um estudo sobre a percepção do micro e pequeno empresário sobre a importância da contabilidade no processo de tomada de decisão. Seminários de Administração FEA-USP, IX, p. 10-11, 2006.

VAN DER VEEKEN, Henk JM; WOUTERS, Marc JF. Using accounting information systems by operations managers in a project company. Management Accounting Research, v. 13, n. 3, p. 345-370, 2002.

VASCONCELOS, Renata Braga Berenguer de; OLIVEIRA, Marcos Roberto Gois de. Determinantes da inovação em micro e pequenas empresas: Uma abordagem gerencial. ERA: Revista de Administração de Empresas, v. 58, n. 4, p. 349-364, 2018.

Artigo recebido em: 15/10/2018

Artigo aprovado em: 26/02/2019

Artigo publicado em: 28/02/2019 